\title{
Atmospheric neutrinos with the first detection units of KM3NeT/ARCA
}

\section{Anna Sinopoulou ${ }^{a, b,{ }^{*}, \text { Rosa Coniglione }}{ }^{c}$, Christos Markou ${ }^{a}$, Rasa Muller ${ }^{d}$, and Ekaterini Tzamariudaki ${ }^{a}$, on behalf of the KM3NeT Collaboration ${ }^{\dagger}$}

a NCSR "Demokritos", Institute of Nuclear and Particle Physics, 15310 Ag. Paraskevi Attikis, Athens, Greece

b National Technical University of Athens, School of Applied Mathematical and Physical Sciences, Zografou Campus, 9, Iroon Polytechniou str, 15780 Zografou, Athens, Greece

c INFN, Laboratori Nazionali del Sud, Via S. Sofia 62, Catania, 95123 Italy

d Nikhef Institute for Subatomic Physics, Science Park 105, 1098 XG Amsterdam, The Netherlands

*E-mail: sinopouloueinp.demokritos.gr

The KM3NeT Collaboration is constructing two deep-sea Cherenkov detectors in the Mediterranean Sea, aiming at neutrino oscillation measurements with the ORCA array, while the ARCA array aims at neutrino astronomy in the TeV range. In April 2021, 5 additional detection units were deployed in the ARCA site. The KM3NeT/ARCA instrumented volume is currently similar to the one of the ANTARES neutrino telescope. In this contribution, an analysis of the data obtained with the detector before April 2021 is presented as well as the analysis of the very first data from the new KM3NeT/ARCA configuration. The performance is demonstrated using atmospheric muons and the first atmospheric neutrinos are shown.

37th International Cosmic Ray Conference (ICRC 2021)

July 12th-23rd, 2021

Online-Berlin, Germany

'The KM3NeT Collaboration authors: The complete list of authors can be found at the end of the proceedings

* Presenter

(c) Copyright owned by the author(s) under the terms of the Creative Commons

Attribution-NonCommercial-NoDerivatives 4.0 International License (CC BY-NC-ND 4.0). 


\section{Introduction}

KM3NeT is a research infrastructure that will host two neutrino detectors: ARCA (Astroparticle Research with Cosmics in the Abyss), located at a depth of $3500 \mathrm{~m}$ offshore Capo Passero (Italy), and ORCA (Oscillation Research with Cosmic in the Abyss), located at a depth of $2450 \mathrm{~m}$ offshore Toulon (France). Their location in the Mediterranean Sea offers an optimal window for the observation of the Southern sky, where the Galactic Centre and most of the Galactic Plane are located. The KM3NeT/ARCA detector, optimised for the neutrino energy range from few $\mathrm{TeV}$ to $100 \mathrm{PeV}$, focuses on high-energy neutrino astrophysics. The KM3NeT/ORCA detector, optimised for neutrino energies from few $\mathrm{GeV}$ to $100 \mathrm{GeV}$, will be dedicated to the study of neutrino oscillations. ARCA and ORCA share the same technology. The main detector component is the digital optical module (DOM), a pressure-resistant glass sphere housing 313 inch photomultiplier tubes (PMTs) and their associated electronics. The lower hemisphere of each DOM contains 19 PMTs, therefore downwards looking, whereas the other 12 PMTs look upwards. The DOMs are arranged in string-like structures, called detection units (DUs), anchored to the seabed, and held vertically by the buoyancy of the DOMs as well as a dedicated buoy at the top. As the two detector designs are optimised for different targeted neutrino energy regimes, they differ in the density of the arrayed photosensors. The vertical spacing between the DOMs along a DU is $36 \mathrm{~m}$ for ARCA and $9 \mathrm{~m}$ for ORCA. The horizontal spacing between the DUs is $\sim 90 \mathrm{~m}$ for ARCA and $\sim 20 \mathrm{~m}$ for ORCA. In their final configurations, ARCA will consist of 230 DUs instrumenting a volume of $\sim 1 \mathrm{~km}^{3}$ of seawater, and ORCA will consist of 115 DUs instrumenting $\sim 7 \mathrm{Mt}$ of seawater. In this contribution, the very first data from the KM3NeT/ARCA configuration will be presented.

\section{Different detector configurations and data taking periods}

The first KM3NeT/ARCA DU was deployed in December 2015. Out of the two additional DUs deployed in May 2016, only one was operational. Therefore, data were collected with two DUs (ARCA2 period). After the commissioning and calibration of ARCA2, a stable and reliable detector performance was achieved. The first data used for analysis were collected on the 23rd of December 2016. Different analyses have been performed in the past concerning ARCA2 as shown in [1],[2]. Due to consecutive upgrades of the seabed network infrastructure, ARCA detector operation was on hold between April 2017 and January 2019, after which, data taking continued with only one operating DU (ARCA1 period). Data from 14 February to 11 November 2019 correspond to this one DU. In mid-April 2021, five more DUs were deployed at the ARCA site and a new data taking period started operating with 6 DUs (ARCA6 period). Once the commissioning and calibration of the detector was carried out, reliable data are being taking steadily. The first data used for analysis were collected on the 12th of May 2021.

The data taking period for ARCA2 in operation has an effective livetime of $\sim 53$ days. The total livetime of the reconstructed data for the second period analysed, ARCA1, is $\sim 207$ days. The atmospheric neutrino candidates presented concern the combined ARCA2 and ARCA1 data taking periods. To demonstrate the performance of the current ARCA configuration, data from the first days of operation with 6 DUs have been reconstructed. The total livetime of ARCA6 data corresponds to $\sim 19$ days. 


\section{Simulation and reconstruction for the atmospheric muon and neutrino samples}

The data were analysed with the standard reconstruction software of KM3NeT [6]. Highquality data (in terms of detector conditions) are selected for all the considered data taking periods. Detailed Monte Carlo (MC) simulations are used to simulate the detector response to atmospheric muons and neutrinos. The simulation chain used for the analyses to be presented in this contribution is similar to the one described in [3], with the addition also of the simulation of the PMT response and the readout as well as of the onshore data filtering. The digitized PMT output pulses are called as hits in the following.

The reconstruction chain applied to simulated events follows the same procedure as for the data. For both atmospheric muon and atmospheric neutrino samples, $\mathrm{MC}$ events were simulated in run-by-run mode which takes into account the time evolution of the data acquisition, in particular the conditions in terms of optical background rates and photon detection efficiencies for each PMT. The PMT photon detection efficiencies are measured and monitored in-situ. The neutrino $\mathrm{MC}$ events were weighted with the atmospheric neutrino flux according to the Honda and Enberg models and a "knee" correction is also applied as described in [3].

Analyses were performed separately for the two detector configurations, ARCA2 and ARCA1 using detailed DATA - MC comparisons and dedicated requirements have been studied and applied to select a clean sample of neutrino candidates. Concerning ARCA6 and due to the limited effective livetime, selection requirements were studied and applied in order to demonstrate the performance of the current configuration in context with the ongoing production plans.

\section{Analysis}

The main goal of ARCA will be the search of astrophysical neutrino sources and the detection of the diffuse astrophysical neutrino flux. For detectors focusing on neutrino astronomy, the background comes mainly from the contribution of atmospheric muons and neutrinos; therefore, the suppression of these contributions, as much as possible, is crucial for cosmic neutrino searches. Currently ARCA is at the construction phase therefore, the instrumented volume is still too small for significant studies in terms of neutrino astronomy. The purpose of this analysis is to demonstrate that with the present configuration of ARCA it is possible to reject the atmospheric muon background and detect atmospheric neutrino candidates. This task is quite challenging for the first ARCA configurations with a low number of DUs, since the final ARCA configuration has been optimised for the detection of high energy neutrinos.

\subsection{Event selection criteria}

As already reported, the main physical background for identifying neutrino candidates comes from the atmospheric muon contribution. Atmospheric muons, produced in CR interactions in the atmosphere, can penetrate to the detector volume if their energy at the sea surface is in the $\mathrm{TeV}$ range or above. The observed rate of the reconstructed atmospheric muons is $0.1 \mathrm{~Hz}$ and 0.2 $\mathrm{Hz}$ for the ARCA1 and ARCA2 configurations respectively, while only 0.2 and 0.5 atmospheric neutrino events per day are expected to be reconstructed, respectively. Atmospheric muons reach 
the detector in a downward direction (from the upper to the down-most part of the detector) as those coming through the Earth interact and are absorbed. Sometimes though, atmospheric muon events are misreconstructed as upward going, particularly when the detector volume is very limited. This analysis aims to reject those misreconstructed atmospheric muon events which constitute a primary step for all the physics analyses in such experiments.

As a first step, detailed comparisons between data and $\mathrm{MC}$ were carried out in order to assess the detector performance. The distribution of the cosine of the reconstructed zenith angle is shown in Fig. 1 for all the reconstructed events for data (black), atmospheric muon simulated events (blue) and atmospheric neutrino simulated events (red) combined for the two detector configurations. As expected, the contribution of the atmospheric muons dominates the observed rate. Atmospheric muon $\mathrm{MC}$ events misreconstructed as upgoing are populating the $\cos \left(\theta_{\text {zenith }}\right)<0$ region. Since atmospheric neutrinos are isotropically distributed, the main goal is to differentiate them from the observed misreconstructed atmospheric muons.

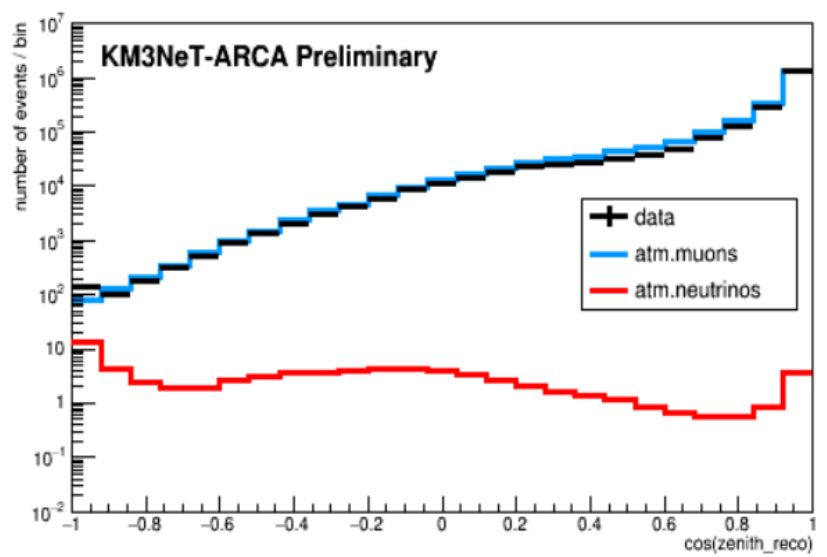

Figure 1. Distribution of the cosine of the zenith angle for all reconstructed events.

In order to do that, the first step is focused on studying and applying requirements to reject poorly reconstructed events. As a second step, dedicated requirements and a cut on the reconstructed zenith angle are applied in order to isolate atmospheric neutrino candidate events. Variables indicating the quality of the track fit are:

- The likelihood $\mathbf{L}$ value of the maximum-likelihood fit. For both configurations, a loose $\mathrm{L}$ requirement is applied to reject misreconstructed events.

- The number of DOMs with signal-like hits. Signal-like hits are those hits with a smalltime residual between the recorded and the expected Cherenkov photon arrival time, i.e., with Cherenkov photons impinging the photocathode surface of the PMT and travelling a small path from the expected emission point along the muon track to the PMT. This requirement suppresses the contribution of background or scattered hits since a sufficient number of DOMs was required to be used for the reconstruction. Well reconstructed events fulfill this condition.

- The track length. Events with longer track lengths are better reconstructed. The track length is calculated as the distance from the reconstructed vertex to the last emission point of a photon with an in-time hit (small time residuals).

- The ratio of the likelihood $L$ value over the number of the hits used for the muon reconstruction Nhits, referred to as $\mathbf{Q}$ or $\mathbf{L} / \mathbf{N h i t s}$, was investigated. In Fig. 2 (left), the distribution 
of the $\mathrm{Q}$ values for upgoing events combining the two detector configurations is shown for data (black), atmospheric muon MC events (blue) and atmospheric neutrino MC events (red), after applying the requirements described before. Applying a cut on $\mathrm{Q}>2.0$, a high rejection of misreconstructed atmospheric muons is achieved while the majority of the atmospheric neutrino events has higher $\mathrm{Q}$ values.
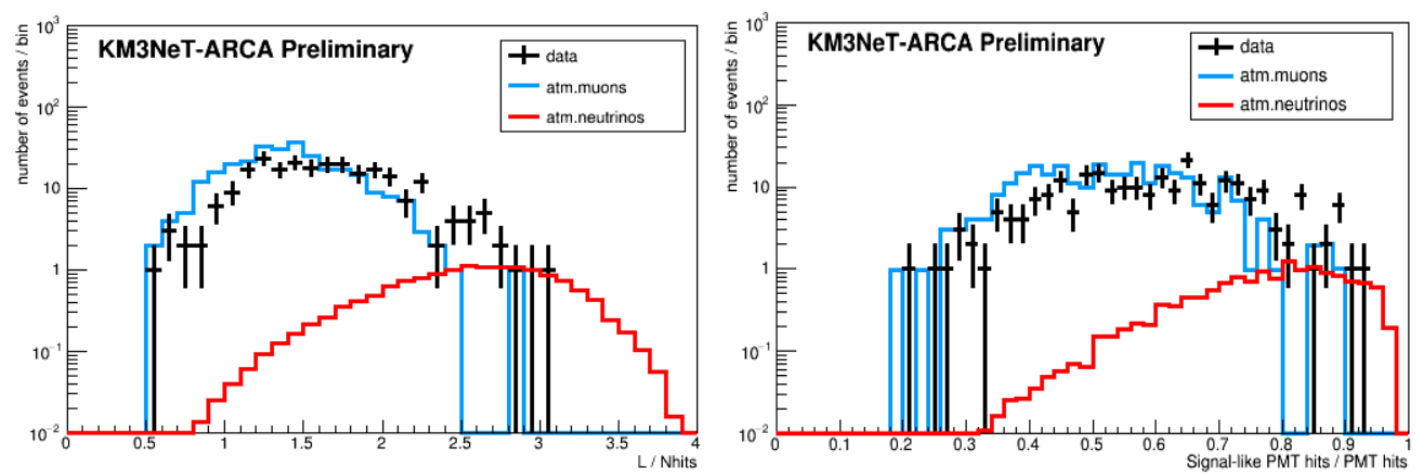

Figure 2. (Left) Distribution of the likelihood over the number of hits used for the muon track reconstruction in an intermediate level of the analysis. (Right) Ratio of the number of PMTs having signal-like hits over all PMTs in an intermediate level of the analysis.

- The ratio of the number of PMTs having signal-like hits over all PMTs used for the track reconstruction. For both detector configurations events were accepted if at least $70 \%$ of the PMTs used for the track reconstruction had signal-like hits. In Fig. 2 (right), the distribution of the ratio is shown for upgoing events combining the two detector configurations for the data (black), atmospheric muon MC (blue) and atmospheric neutrino MC (red) after the previously stated requirements are applied. One can notice that misreconstructed atmospheric muon events, after applying the requirements, have lower numbers of PMTs with signal-like hits compared to atmospheric neutrino events.

\subsection{Search for atmospheric neutrinos}

The final step of the analysis is focused on identifying upgoing atmospheric neutrino events. As mentioned before, a contribution from misreconstructed atmospheric muons could remain in the final sample of events. In order to reduce this contamination, two additional requirements to the final sample for both configurations are investigated:

- The containment requirement. Tracks with an upward going direction usually have their reconstructed vertex at the lower half of the detector. An investigation of the upward going atmospheric neutrino MC events was carried out in order to set the maximum accepted height of the reconstructed vertex. No cut has been applied for the radial distance of the vertex from the DU.

- The time residuals requirement. The time residuals between the recorded and the expected Cherenkov photon arrival time are investigated since, larger residuals are expected for misreconstructed atmospheric muons comparing to the atmospheric neutrino MC. Therefore, a time window was set in order to isolate neutrino events. 


\section{Detection of atmospheric neutrinos for ARCA1\&ARCA2}

The distribution of the cosine of the reconstructed zenith angle is shown in Fig. 3 for all the events surviving the selection criteria. ARCA1 and ARCA2 operation periods have been combined for data (black), atmospheric muon MC (blue) and atmospheric neutrino MC (red). A powerful rejection of atmospheric muons misreconstructed as upgoing has been achieved as only one atmospheric muon event reconstructed with an upgoing track direction survives the final selection. A total number of 15 neutrino candidates have been found with $\cos \left(\theta_{\text {zenith }}\right)<0$, while 8.1 is expected from the atmospheric neutrino MC sample in $\sim 260$ days of total livetime.

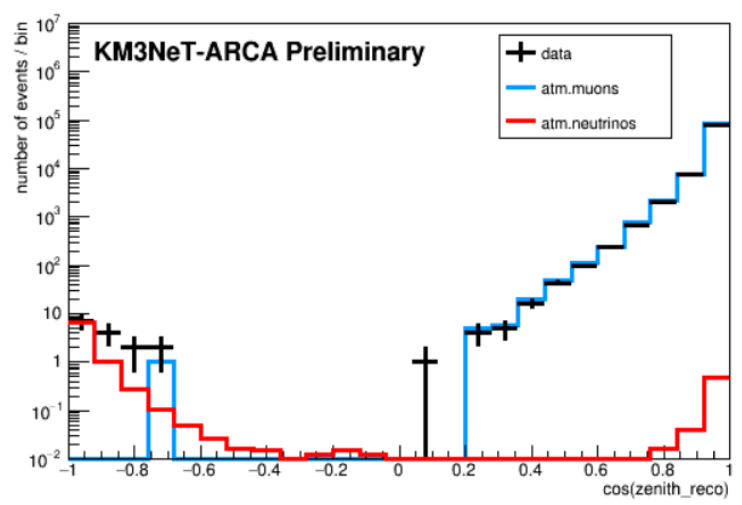

Figure 3. Distribution of the cosine of the zenith angle for events surviving the neutrino selection criteria.

An example of an event display is shown as recorded in Fig. 4, where the height of the DOM hit is shown as a function of the measured hit time. The height is given with respect to the lowest DOM. All DOMs with hits are represented by blue circles while DOMs with triggered hits are shown with red circles. The magenta dotted line shows, for direct Cherenkov photons, the arrival time to the DOM as expected from the reconstructed muon track.

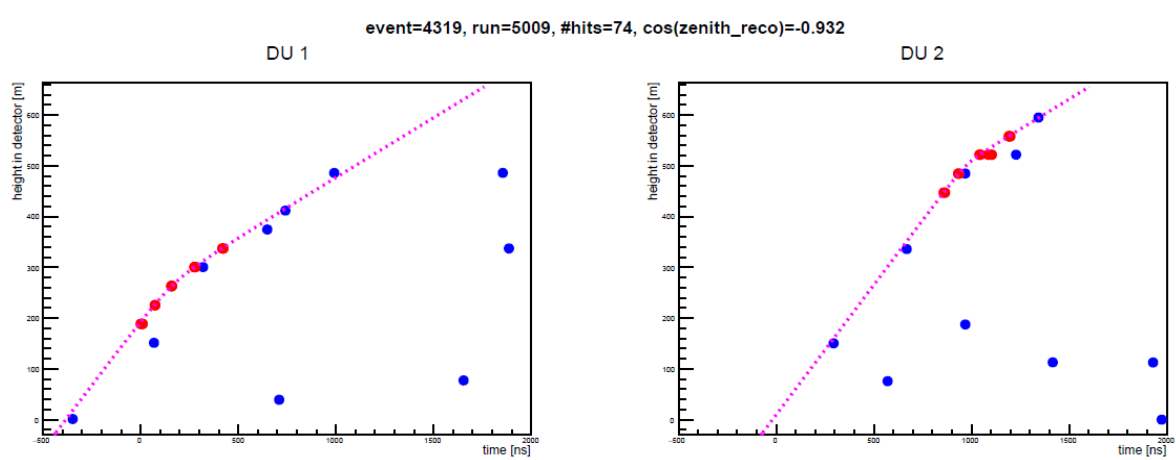

Figure 4. Height in the detector vs time of the recorded PMT hits for a selected event in ARCA2 configuration.

\section{Current ARCA configuration - ARCA6}

ARCA is currently operating with 6 DUs with stable data taking since the middle of May 2021. The data analysed in this contribution have an effective livetime of $\sim 19$ days. The distribution of the cosine of the reconstructed zenith angle is shown in Fig. 5 (left) for all the reconstructed events for data (black), atmospheric muon simulated events (blue) and atmospheric neutrino simulated events (red). The requirements to reject poorly reconstructed events are based on detailed Data/MC comparisons. The variables used to characterise the quality of the track fit 
are similar to those used for the ARCA1\&ARCA2 analysis. In addition, a requirement on the maximum accepted angular error of the track reconstruction fit was investigated and applied.

After applying all the requirements, the distribution of the cosine of the reconstructed zenith angle is shown in Fig. 5 (right) for all the reconstructed events for data (black), atmospheric muon simulated events (blue) and atmospheric neutrino simulated events (red).
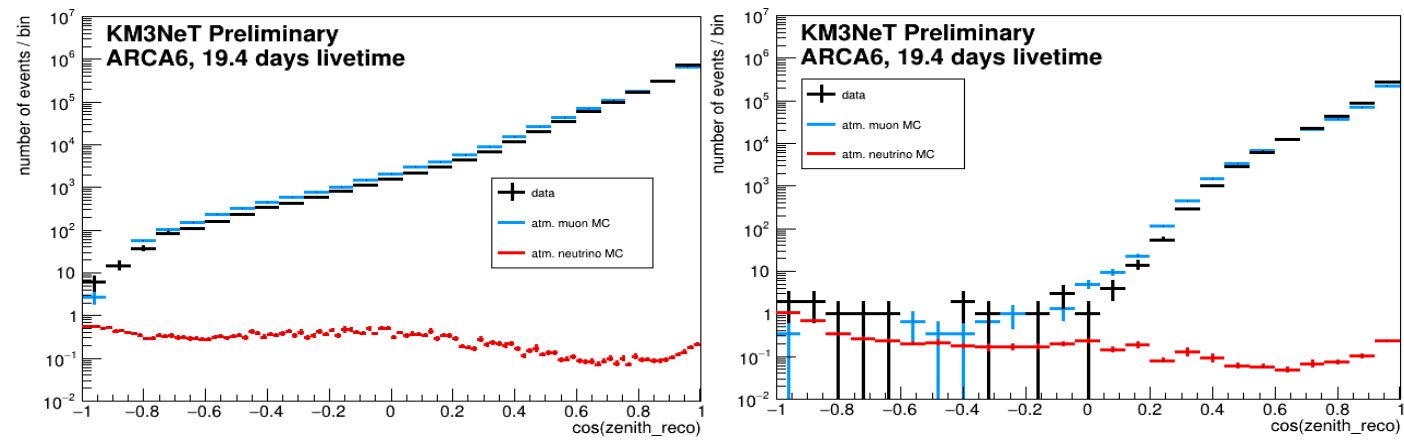

Figure 5. Distribution of the cosine of the zenith angle for all reconstructed events (left) and for events surviving the neutrino selection criteria (right).

A powerful rejection of atmospheric muons misreconstructed as upgoing events has been achieved. A total number of 15 upgoing events are observed with an upgoing track direction while 7 events are expected from the atmospheric muon and 4 events from the atmospheric neutrino simulation. Focusing on the upgoing track direction with $\cos \left(\theta_{\text {zenith }}\right)<-0.8,5$ events are observed while 2 are expected from the atmospheric neutrino simulation without any contribution from the atmospheric muons.

For all the reconstructed upgoing neutrino $\mathrm{MC}$ events in the low energy range ( $E<10 \mathrm{TeV}$ ) ARCA6 is expected to have an effective area comparable to the current ORCA configuration (6 operational DUs - ORCA6) and the ANTARES telescope. For the high energy range $(E>$ $100 \mathrm{TeV}$ ), for which ARCA is optimised, ARCA6 is expected to perform significantly better than ANTARES as shown in Fig. 6 (left).

An excellent angular resolution of less than $0.1^{\circ}$ for $E>100 \mathrm{TeV}$ for the full ARCA detector is expected, due to the innovative multi-PMT DOMs of KM3NeT and the large scattering length of deep-sea water. For the current ARCA configuration, the angular resolution is shown after the quality selection requirements in Fig. 6 (right) as a function of the true (MC) neutrino energy. The median of the angular resolution is $0.75^{\circ}$.
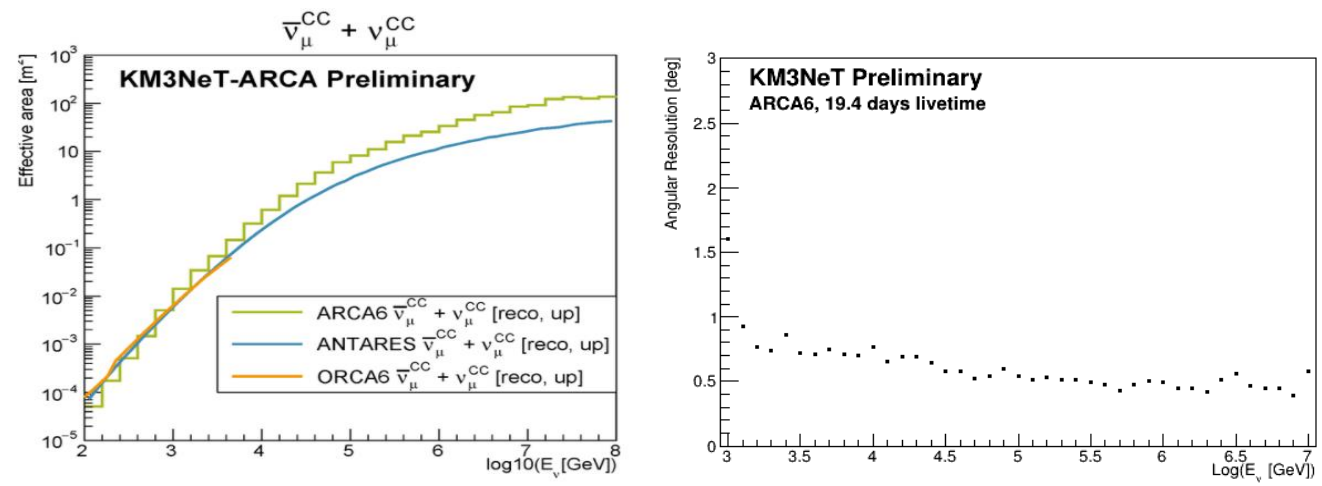

Figure 6. (Left) Effective area for all neutrino MC events reconstructed as upgoing for ARCA6 (green), ANTARES (blue) and ORCA6 (orange). (Right) Angular resolution for events surviving the selection criteria as a function of the true neutrino energy. 


\section{Conclusion and outlook}

For the past and current ARCA detector configurations, the search for atmospheric neutrino candidates is more challenging than for ORCA due to the higher neutrino energy detection threshold, the very limited detector volume and the small effective livetime of ARCA. Within a period of $\sim 260$ days of effective livetime, with one (ARCA1) and two (ARCA2) operational DUs, 15 neutrino candidates have been observed, with one atmospheric muon expected from the MC surviving the final selection criteria. The data are in a reasonable agreement with the MC simulation before and after applying the selection requirements.

With the current ARCA configuration of 6 operational DUs and an effective livetime of only 19 days, 15 events were observed as upward going events while 4 are expected from the atmospheric neutrino simulation and 9 from the atmospheric muon simulation. Also in this case, data and Monte Carlo show a reasonable agreement before and after selection criteria is applied. The median of the angular resolution after the event selection is $0.75^{\circ}$. For the low energy range $(E<10 \mathrm{TeV})$ ARCA6 is expected to have comparable effective area to the current ORCA configuration (ORCA6) and to ANTARES. In the energy range of interest for ARCA ( $E>$ $100 \mathrm{TeV}$ ), ARCA6 is expected to perform significantly better than ANTARES.

The aim of this analysis has been to show that with a $1-3 \%$ of the full instrumented volume of the ARCA detector, it is possible to detect atmospheric neutrinos and to achieve a powerful reduction of the atmospheric muon contribution. Furthermore, a good data/MC agreement verifies the KM3NeT technology, the detector understanding and detector calibration demonstrating the capability of the future $\mathrm{KM} 3 \mathrm{NeT}$ detectors.

\section{Acknowledgements}

Anna Sinopoulou acknowledges the support of the Hellenic Foundation for Research and Innovation (H.F.R.I.) under the HFRI PhD Fellowship grant (Fellowship Number (1076)).

\section{H.F.R.I}

\section{References}

[1] "Detection of atmospheric neutrinos with the first detection units of KM3NeT-ARCA", Anna Sinopoulou*, Rosa Coniglione, Ekaterini Tzamariudaki, for the KM3NeT Collaboration, XXIX INTERNATIONAL CONFERENCE ON NEUTRINO PHYSICS - Neutrino2020

[2] "Atmospheric Neutrinos Detected with the First KM3NeT Detection Units of ARCA and ORCA", Rosa Coniglione, Jannik Hofestädt †, Anna Sinopoulou, Ekaterini Tzamariudaki, Dmitry Zaborov on behalf of the KM3NeT collaboration, PoS(ICRC2019)910, 36th International Cosmic Ray Conference -ICRC 2019

[3] S. Adrián-Martínez et al. (KM3NeT Collaboration), 2016 J. Phys. G 43084001

[4] M. Ageron et al. (KM3NeT Collaboration), arXiv:1906.02704 [physics.ins-det]

[5] M. Honda et al., Phys. Rev. D 92, 023004 (2015)

[6] K.Melis, A. Heijboer, M. de Jong, on behalf of the KM3NeT Collaboration, PoS(ICRC2017)950, 35th International Cosmic Ray Conference -ICRC2017 


\section{Full Authors List: KM3NeT Collaboration}

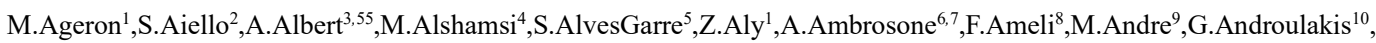
M. Anghinolfi ${ }^{11}$, M. Anguita ${ }^{12}$, G. Anton ${ }^{13}$, M. $\operatorname{Ardid}^{14}$, S. $\operatorname{Ardid}^{14}$, W. Assal ${ }^{1}$, J. Aublin ${ }^{4}$, C. Bagatelas ${ }^{10}$, B. Baret ${ }^{4}$, S. Basegmez du Pree $^{15}$, M. Bendahman ${ }^{4,16}$, F. Benfenati ${ }^{17,18}$, E. Berbee ${ }^{15}$, A.M. van den Berg ${ }^{19}$, V. Bertin ${ }^{1}$, S. Beurthey ${ }^{1}$, V. van Beveren ${ }^{15}$, S. Biagi ${ }^{20}$, M. Billault ${ }^{1}$, M. Bissinger ${ }^{13}$, M. Boettcher ${ }^{21}$, M. Bou Cabo ${ }^{22}$, J. Boumaaza ${ }^{16}$, M. Bouta ${ }^{23}$, C. Boutonnet ${ }^{4}$, G. Bouvet ${ }^{24}$, M Bouwhuis $^{15}$, C. Bozza ${ }^{25}$, H.Brânzaş ${ }^{26}$, R. Bruijn ${ }^{15,27}$, J. Brunner ${ }^{1}$, R. Bruno ${ }^{2}$, E. Buis ${ }^{28}$, R. Buompane ${ }^{6,29}$, J. Busto ${ }^{1}$, B. Caiffi ${ }^{11}$, Caillat $^{1}$, D. Calvo ${ }^{5}$, S. Campion ${ }^{30,8}$, A. Capone ${ }^{30,8}$, H. Carduner ${ }^{24}$, V. Carretero ${ }^{5}$, P. Castaldi ${ }^{17,31}$, S. Celli ${ }^{30,8}$, R. Cereseto ${ }^{11}$, Chabab ${ }^{32}$, C. Champion ${ }^{4}$, N. Chau ${ }^{4}$, A. Chen ${ }^{33}$, S. Cherubini ${ }^{20,34}$, V. Chiarella ${ }^{35}$, T. Chiarusi ${ }^{17}$, M. Circella ${ }^{36}$, R. Cocimano ${ }^{20}$, J.A.B. Coelho ${ }^{4}$, A. Coleiro ${ }^{4}$, M. Colomer Molla ${ }^{4,5}$, S. Colonges ${ }^{4}$, R. Coniglione ${ }^{20}$, A. Cosquer ${ }^{1}$, P. Coyle $^{1}$, M. Cresta $^{11}$, A. Creusot ${ }^{4}$, A. Cruz ${ }^{37}$, G. Cuttone $^{20}$, A. D’Amico ${ }^{15}$, R. Dallier ${ }^{24}$, B. De Martino ${ }^{1}$, M. De Palma ${ }^{36,38}$, I. Di Palma ${ }^{30,8}$, A.F. Díaz ${ }^{12}$, D. Diego-Tortosa ${ }^{14}$, C. Distefano $^{20}$, A. Domi ${ }^{15,27}$, C. Donzaud ${ }^{4}$, D. Dornic ${ }^{1}$, M. Dörr ${ }^{39}$, D. Drouhin ${ }^{3,55}$, T. Eberl ${ }^{13}$, A. Eddyamoui ${ }^{16}$, T. van Eeden ${ }^{15}$, D. van Eijk $^{15}$, I. El Bojaddaini23, H. Eljarrari ${ }^{16}$, D. Elsaesser ${ }^{39}$, A. Enzenhöfer ${ }^{1}$, V. Espinosa ${ }^{14}$, P. Fermani ${ }^{30,8}$, G. Ferrara ${ }^{20,34}$, M. D. Filipović $^{40}$, F. Filippini ${ }^{17,18}$, J. Fransen ${ }^{15}$, L.A. Fusco ${ }^{1}$, D. Gajanana ${ }^{15}$, T. Gal ${ }^{13}$, J. García Méndez ${ }^{14}$, A. Garcia Soto ${ }^{5}$, E. Garçon ${ }^{1}$, F. Garufi $^{6,7}$, C. Gatius ${ }^{15}$, N. Geißelbrecht ${ }^{13}$, L. Gialanella ${ }^{6,29}$, E. Giorgio ${ }^{20}$, S.R. Gozzini ${ }^{5}$, R. Gracia ${ }^{15}$, K. Graf ${ }^{13}$, G. Grella ${ }^{41}$, D

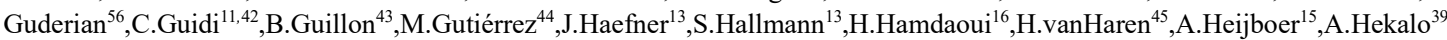
,L.Hennig ${ }^{13}$,S.Henry ${ }^{1}$,J.J.Hernández-Rey ${ }^{5}$,J.Hofestädt ${ }^{13}$,F.Huang ${ }^{1}$,W.Idrissilbnsalih ${ }^{6,29}$, A. Ilioni ${ }^{4}$, G. Illuminati ${ }^{17,18,4}$, C.W. James ${ }^{37}$, D.Janezashvili ${ }^{46}$,P.Jansweijer ${ }^{15}$,M.deJong ${ }^{15,47}$,P.deJong ${ }^{15,27}$,B.J.Jung ${ }^{15}$,M.Kadler ${ }^{39}$,P.Kalaczyński ${ }^{48}$,O.Kalekin ${ }^{13}$,U.F.Katz ${ }^{13}$,F.Kayzel ${ }^{15}$,P.Keller ${ }^{1}$,N.R.KhanChowdhury ${ }^{5}$,G.Kistauri ${ }^{46}$,F.vanderKnaap ${ }^{28}$, P. Kooijman ${ }^{27,57}$, A. Kouchner ${ }^{4,49}$, M. Kreter ${ }^{21}$, V. Kulikovskiy ${ }^{11}$, M. Labalme ${ }^{43}$, P. Lagier ${ }^{1}$, R. Lahmann ${ }^{13}$, P. Lamare ${ }^{1}$, M. Lamoureux $1^{4}$, G. Larosa ${ }^{20}$, C. Lastoria ${ }^{1}$, J. Laurence ${ }^{1}$, A. Lazo ${ }^{5}$, R. Le Breton $^{4}$, E. Le Guirriec ${ }^{1}$, S. Le Stum ${ }^{1}$, G. Lehaut ${ }^{43}$, O. Leonardi ${ }^{20}$, F. Leone ${ }^{20,34}$, E. Leonora ${ }^{2}$, C. Lerouvillois ${ }^{1}$, J. Lesrel ${ }^{4}$, N. Lessing $^{13}$, G. Levi ${ }^{17,18}$, M. Lincetto ${ }^{1}$, M. Lindsey Clark ${ }^{4}$, T. Lipreau ${ }^{24}$, C. LLorens Alvarez ${ }^{14}$, A. Lonardo ${ }^{8}$, F. Longhitano ${ }^{2}$, D. LopezCoto $^{44}$, N. Lumb ${ }^{1}$, L. Maderer ${ }^{4}$, J. Majumdar ${ }^{15}$, J. Mańczak ${ }^{5}$, A. Margiotta ${ }^{17,18}$, A. Marinelli ${ }^{6}$, A. Marini ${ }^{1}$, C. Markou ${ }^{10}$, L. Martin $^{24}$, J.A.MartínezMora ${ }^{14}$,A.Martini ${ }^{35}$,F.Marzaioli ${ }^{6,29}$,S.Mastroianni ${ }^{6}$,K.W.Melis ${ }^{15}$, G.Miele $^{6,7}$, P.Migliozzi ${ }^{6}$,E.Migneco ${ }^{20}$,P.Mijakowski ${ }^{48}$,

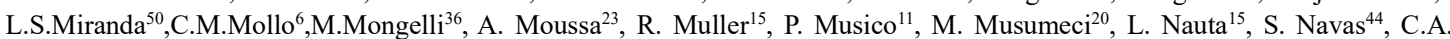
$\mathrm{Nicolau}^{8}$, B. Nkosi ${ }^{33}$, B. Ó Fearraigh ${ }^{15,27}$, M. O'Sullivan ${ }^{37}$, A. Orlando ${ }^{20}$, G. Ottonello ${ }^{11}$, S. Ottonello ${ }^{11}$, J. Palacios González ${ }^{5}$, G. Papalashvili $^{46}$, R. Papaleo ${ }^{20}$, C. Pastore ${ }^{36}$, A. M. Păun ${ }^{26}$, G.E. Păvălaş ${ }^{26}$, G. Pellegrini ${ }^{17}$, C. Pellegrino ${ }^{18,58}$, M. Perrin-Terrin ${ }^{1}$, V. Pestel $^{15}$, P. Piattelli ${ }^{20}$, C. Pieterse ${ }^{5}$, O. Pisanti ${ }^{6,7}$, C. Poire ${ }^{14}$, V. Popa $^{26}$, T. Pradier ${ }^{3}$, F. Pratolongo ${ }^{11}$, I. Probst ${ }^{13}$, G. Pühlhofer ${ }^{51}$, S. Pulvirenti $^{20}$, G. Quéméner ${ }^{43}$, N. Randazzo ${ }^{2}$, A. Rapicavoli ${ }^{34}$, S. Razzaque ${ }^{50}$, D. Real ${ }^{5}$, S. Reck ${ }^{13}$, G. Riccobene ${ }^{20}$, L. Rigalleau ${ }^{24}$, A Romanov $^{11,42}$, A. Rovellii ${ }^{20}$, J. Royon ${ }^{1}$, F. Salesa Greus ${ }^{5}$, D.F.E. Samtleben ${ }^{15,47}$, A. Sánchez Losa ${ }^{36,5}$, M. Sanguineti ${ }^{11,42}$, A Santangelo $^{51}$, D. Santonocito ${ }^{20}$, P.Sapienza ${ }^{20}$, J.Schmelling ${ }^{15}$, J.Schnabel ${ }^{13}$, M.F.Schneider ${ }^{13}$, J.Schumann ${ }^{13}$, H.M.Schutte ${ }^{21}$,

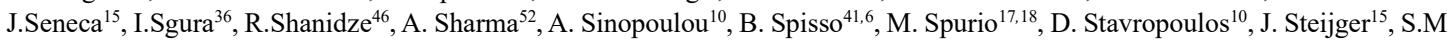
Stellacci ${ }^{41,6}$, M. Taiuti ${ }^{11,42}$, F. Tatone ${ }^{36}$, Y. Tayalati ${ }^{16}$, E. Tenllado ${ }^{44}$, D. Tézier ${ }^{1}$, T. Thakore ${ }^{5}$, S. Theraube ${ }^{1}$, H. Thiersen ${ }^{21}$, P. Timmer ${ }^{15}$, S. Tingay ${ }^{37}$, S. Tsagkli ${ }^{10}$, V. Tsourapis ${ }^{10}$, E. Tzamariudaki ${ }^{10}$, D. Tzanetatos ${ }^{10}$, C. Valieri ${ }^{17}$, V. Van Elewyck ${ }^{4,49}$, G. Vasileiadis ${ }^{53}$, F. Versari $^{17,18}$, S. Viola ${ }^{20}$, D. Vivolo ${ }^{6,29}$, G. de Wasseige ${ }^{4}$, J. Wilms ${ }^{54}$, R. Wojaczyński ${ }^{48}$, E. de Wolf ${ }^{15,27}$, T. Yousfi ${ }^{23}$, S. Zavatarelli ${ }^{11}$, A. Zegarelli $^{30,8}$, D. Zito ${ }^{20}$, J.D. Zornoza ${ }^{5}$, J. Zúñiga ${ }^{5}$, N. Zywucka ${ }^{21}$.

${ }^{1}$ Aix Marseille Univ, CNRS/IN2P3, CPPM, Marseille, France.

${ }^{2}$ INFN, Sezione di Catania, Via Santa Sofia 64, Catania, 95123 Italy.

${ }^{3}$ Université de Strasbourg, CNRS, IPHC UMR 7178, F-67000 Strasbourg, France.

${ }^{4}$ Université de Paris, CNRS, Astroparticule et Cosmologie, F-75013 Paris, France.

${ }^{5}$ IFIC - Instituto de Física Corpuscular (CSIC - Universitat de València), c/Catedrático José Beltrán, 2, 46980 Paterna, Valencia, Spain. ${ }^{6}$ INFN, Sezione di Napoli, Complesso Universitario di Monte S. Angelo, Via Cintia ed. G, Napoli, 80126 Italy.

${ }^{7}$ Università di Napoli “Federico II”, Dip. Scienze Fisiche “E. Pancini”, Complesso Universitario di Monte S. Angelo, Via Cintia ed. G, Napoli, 80126 Italy.

${ }^{8}$ INFN, Sezione di Roma, Piazzale Aldo Moro 2, Roma, 00185 Italy.

${ }^{9}$ Universitat Politècnica de Catalunya, Laboratori d'Aplicacions Bioacústiques, Centre Tecnològic de Vilanova i la Geltrú, Avda. Rambla Exposició, s/n, Vilanova i la Geltrú, 08800 Spain.

${ }^{10}$ NCSR Demokritos, Institute of Nuclear and Particle Physics, Ag. Paraskevi Attikis, Athens, 15310 Greece.

${ }^{11}$ INFN, Sezione di Genova, Via Dodecaneso 33, Genova, 16146 Italy.

${ }^{12}$ University of Granada, Dept. of Computer Architecture and Technology/CITIC, 18071 Granada, Spain.

${ }^{13}$ Friedrich-Alexander-Universität Erlangen-Nürnberg, Erlangen Centre for Astroparticle Physics, Erwin-Rommel-Straße 1, 91058 Erlangen, Germany.

${ }^{14}$ Universitat Politècnica de València, Instituto de Investigación para la Gestión Integrada de las Zonas Costeras, C/ Paranimf, 1, Gandia, 46730 Spain.

${ }^{15}$ Nikhef, National Institute for Subatomic Physics, PO Box 41882, Amsterdam, 1009 DB Netherlands.

${ }^{16}$ University Mohammed V in Rabat, Faculty of Sciences, 4 av. Ibn Battouta, B.P. 1014, R.P. 10000 Rabat, Morocco.

${ }^{17}$ INFN, Sezione di Bologna, v.le C. Berti-Pichat, 6/2, Bologna, 40127 Italy.

${ }^{18}$ Università di Bologna, Dipartimento di Fisica e Astronomia, v.le C. Berti-Pichat, 6/2, Bologna, 40127 Italy.

${ }^{19} \mathrm{KVI}$-CART University of Groningen, Groningen, the Netherlands. 
${ }^{20}$ INFN, Laboratori Nazionali del Sud, Via S. Sofia 62, Catania, 95123 Italy.

${ }^{21}$ North-West University, Centre for Space Research, Private Bag X6001, Potchefstroom, 2520 South Africa.

${ }^{22}$ Instituto Español de Oceanografía, Unidad Mixta IEO-UPV, C/ Paranimf, 1, Gandia, 46730 Spain.

${ }^{23}$ University Mohammed I, Faculty of Sciences, BV Mohammed VI, B.P. 717, R.P. 60000 Oujda, Morocco.

${ }^{24}$ Subatech, IMT Atlantique, IN2P3-CNRS, Université de Nantes, 4 rue Alfred Kastler - La Chantrerie, Nantes, BP 2072244307

France. ${ }^{25}$ Università di Salerno e INFN Gruppo Collegato di Salerno, Dipartimento di Matematica, Via Giovanni Paolo II 132, Fisciano, 84084

Italy.

${ }^{26}$ ISS, Atomistilor 409, Măgurele, RO-077125 Romania.

${ }^{27}$ University of Amsterdam, Institute of Physics/IHEF, PO Box 94216, Amsterdam, 1090 GE Netherlands.

${ }^{28}$ TNO, Technical Sciences, PO Box 155, Delft, 2600 AD Netherlands.

${ }^{29}$ Università degli Studi della Campania "Luigi Vanvitelli", Dipartimento di Matematica e Fisica, viale Lincoln 5, Caserta, 81100 Italy. ${ }^{30}$ Università La Sapienza, Dipartimento di Fisica, Piazzale Aldo Moro 2, Roma, 00185 Italy.

${ }^{31}$ Università di Bologna,Dipartimento di Ingegneria dell' Energia Elettrica e dell' Informazione "GuglielmoMarconi", Via dell' Università 50, Cesena, 47521 Italia.

${ }^{32}$ Cadi Ayyad University, Physics Department, Faculty of Science Semlalia, Av. My Abdellah, P.O.B. 2390, Marrakech, 40000 Morocco.

${ }^{33}$ University of the Witwatersrand, School of Physics, Private Bag 3, Johannesburg, Wits 2050 South Africa.

${ }^{34}$ Università di Catania, Dipartimento di Fisica e Astronomia "Ettore Majorana", Via Santa Sofia 64, Catania, 95123 Italy.

${ }^{35}$ INFN, LNF, Via Enrico Fermi, 40, Frascati, 00044 Italy.

${ }^{36}$ INFN, Sezione di Bari, via Orabona, 4, Bari, 70125 Italy.

${ }^{37}$ International Centre for Radio Astronomy Research, Curtin University, Bentley, WA 6102, Australia.

${ }^{38}$ University of Bari, Via Amendola 173, Bari, 70126 Italy.

${ }^{39}$ University Würzburg, Emil-Fischer-Straße 31, Würzburg, 97074 Germany.

${ }^{40}$ Western Sydney University, School of Computing, Engineering and Mathematics, Locked Bag 1797, Penrith, NSW 2751 Australia.

${ }^{41}$ Università di Salerno e INFN Gruppo Collegato di Salerno, Dipartimento di Fisica, Via Giovanni Paolo II 132, Fisciano, 84084 Italy.

${ }^{42}$ Università di Genova, Via Dodecaneso 33, Genova, 16146 Italy.

${ }^{43}$ Normandie Univ, ENSICAEN, UNICAEN, CNRS/IN2P3, LPC Caen, LPCCAEN, 6 boulevard Maréchal Juin, Caen, 14050 France.

${ }^{44}$ University of Granada, Dpto. de Física Teórica y del Cosmos \& C.A.F.P.E., 18071 Granada, Spain.

${ }^{45} \mathrm{NIOZ}$ (Royal Netherlands Institute for Sea Research), PO Box 59, Den Burg, Texel, 1790 AB, the Netherlands.

${ }^{46}$ Tbilisi State University, Department of Physics, 3, Chavchavadze Ave., Tbilisi, 0179 Georgia.

${ }^{47}$ Leiden University, Leiden Institute of Physics, PO Box 9504, Leiden, 2300 RA Netherlands.

${ }^{48}$ National Centre for Nuclear Research, 02-093 Warsaw, Poland.

${ }^{49}$ Institut Universitaire de France, 1 rue Descartes, Paris, 75005 France.

${ }^{50}$ University of Johannesburg, Department Physics, PO Box 524, Auckland Park, 2006 South Africa.

${ }^{51}$ Eberhard Karls Universität Tübingen, Institut für Astronomie und Astrophysik, Sand 1, Tübingen, 72076 Germany.

${ }^{52}$ Università di Pisa, Dipartimento di Fisica, Largo Bruno Pontecorvo 3, Pisa, 56127 Italy.

${ }^{53}$ Laboratoire Univers et Particules de Montpellier, Place Eugène Bataillon - CC 72, Montpellier Cédex 05, 34095 France.

${ }^{54}$ Friedrich-Alexander-Universität Erlangen-Nürnberg, Remeis Sternwarte, Sternwartstraße 7, 96049 Bamberg, Germany.

${ }^{55}$ Université de Haute Alsace, 68100 Mulhouse Cedex, France.

${ }^{56}$ University of Münster, Institut für Kernphysik, Wilhelm-Klemm-Str. 9, Münster, 48149 Germany.

${ }^{57}$ Utrecht University, Department of Physics and Astronomy, PO Box 80000, Utrecht, 3508 TA Netherlands.

${ }^{58}$ INFN, CNAF, v.le C. Berti-Pichat, 6/2, Bologna, 40127 Italy. 